\title{
ASSESSING DRIVER FITNESS TO PARTICIPATE IN FHWA FIELD EXPERIMENTATION AT NIGHT
}

\author{
John A. Molino ${ }^{1}$, Kenneth S. Opiela ${ }^{2}$, \\ Bryan J. Katz ${ }^{1}$, M. Joseph Moyer ${ }^{2}$ \\ ${ }^{1}$ Science Applications International Corporation \\ ${ }^{2}$ Federal Highway Administration \\ FHWA Turner Fairbank Highway Research Center \\ McLean, Virginia, USA \\ E-mail: John.A.Molino@,saic.com
}

\begin{abstract}
Summary: A Driving Fitness Form was developed to ensure that drivers are ready to participate in field driving experiments at night. The form was tested in a field experiment conducted in Delta, Pennsylvania, during August 2004. The field experiment was part of a cooperative research program conducted by the Federal Highway Administration and the Pennsylvania Department of Transportation. Sixteen research participants drove a curvy stretch of rural two-lane highway each night for 8 nights with different pavement markings and markers on the roadway. Partly due to the Driving Fitness Form, the experiment was completed successfully by all participants without any incidents or crashes. The Driving Fitness Form performed well, and summary data were collected on the sample of 16 drivers.
\end{abstract}

\section{OBJECTIVES}

The Federal Highway Administration (FHWA) is conducting a research program on the effects of roadway delineation (pavement markings and markers) to provide guidance to drivers at night. As a part of this research program, field experiments are conducted in which research participants drive an FHWA instrumented vehicle on curvy roads at night. It is essential to assess the driving fitness of each research participant on each night that test drives are made. The objective of this part of the research was to develop an assessment instrument for fitness to drive, to test this instrument in a field experiment, to collect data on a sample of research participants, and to characterize the sample of participants in terms of variables important to driving safely at night. The instrument was developed by extracting important concepts from the literature (e.g., Hartley, 1995; NIOSH1, 1997), and from previous FHWA experience with nighttime driving experiments.

\section{METHOD}

The primary method used in the reported field experiment consisted of a Driving Fitness Form. In the actual experiment, this form was called the "Fatigue Assessment Form," although some of the questions inquired about factors other than fatigue (see Appendix). Future versions of this form will be called the "Driving Fitness Form," and that is the name used throughout the present paper.

Before the experiment began, the research participants were told that they had to be awake and alert to drive each night. The Driving Fitness Form was administered each night before each test 
drive. As can be seen in the Appendix, the form asks 14 basic questions about the state of fatigue and condition of alertness for driving in a nighttime field experiment. The questions concern the time, length and quality of the last night's sleep, the hours, length and regularity of recent work activity, and prescription medicine, alcohol and other substance intake over the past 24 hours. The experimenter reviewed this Form each night before authorizing the particular research participant to commence the scheduled test drive. If some item stood out, like alcohol consumption in the last 12 hours, or less than 4 hours of sleep, the experimenter probed the situation more deeply with the participant. If there were a satisfactory explanation, the test drive was permitted. If not, the participant did not drive that night, and was requested to return the next night. The experimenter also made a visual and conversational assessment of the condition of each driver each night, and was vigilant for any unusual driving behaviors once the test drive had begun.

Although they did not drive, both the experimenter and the safety observer who rode in the test vehicle also filled out the Driving Fitness Form each night, since they were responsible for the overall safety of the experiment. Both the experimenter and the safety observer were required to maintain a consistent shift-work schedule for the entire duration of the experiment, including a 3day adaptation period before any research participants were run. They both went to bed around 7:00 AM and woke up around 3:00 PM every day for a period of about 15 consecutive days, even on their non-working days. In order to enforce this shift-work pattern, the experiment protocol called for them not to leave the site for the entire 15-day period.

\section{OVERALL RESULTS}

The Driving Fitness Form was employed for the first time in the FHWA field experiment described above. This experiment was conducted in cooperation with the Pennsylvania Department of Transportation. The Form was approved by the SAIC Institutional Review Board (IRB) for use in that study. The study was conducted with 16 research participants who drove a 3-mile stretch of curvy roadway in rural Pennsylvania for 8 consecutive nights in August 2004. Experimental drives were scheduled each half hour from 9:00 PM to 5:00 AM the next morning. Each participant drove at the same time each night, with only a few minor exceptions. No experimental drives were conducted during rain or with a wet roadway. Half of the research participants were younger drivers (18 to 26 years old) and half were older drivers (61 to 79 years old). Half were males and half were females. Each participant was paid \$240 upon completion of the experiment. Altogether 128 drives should have been completed (16 participants $\mathrm{x} 8$ nights). One participant did not complete one drive, so only 127 Forms were filled out. Before each drive, the Driving Fitness Form was administered. No drivers were rejected for the night's drive based upon information provided on the form or upon other observations made by the experimenter. One Form was lost after the experiment was over, so the data presented are for 126 responses. The responses of the experimenter and the safety observer were not analyzed in the present paper.

The Driving Assessment Form reproduced in the Appendix is how the actual form looked, except that statistical results have been inserted in brackets in the blanks. The data in the brackets characterize the particular sample of 16 participants and 126 drives reported, according to the measured dimensions of fitness to drive in the experiment. As can be seen on the Form, during the period of the experiment (about 12 total days with 4 days missed because of weather), the 
entire sample slept an average of 6.62 hours per night, with a standard deviation of 2.2 hours. They awoke at an average time of 7:59 AM. The average time for going to bed was an artifact of the experimental regime, where people came in to drive at all hours of the night. Thus the average bedtime of the sample is designated as "not applicable" in brackets on the Form. Their quality of sleep was generally "good" $(62 \%)$ and their sleep pattern was generally not "regular" $(63 \%)$. This latter result is to be expected, since the participatants got up to drive at all hours of the night. In this particular sample, 48\% worked and 52\% did not work. Of those who worked, the average starting time for work was 11:03 AM, and the average ending time was 4:13 PM, with $22 \%$ representing shift workers. The presence of shift workers and the unusual hours of experimentation may have distorted the work pattern data to some extent. No participant had crossed any time zones in the previous 3 days. For the particular sample of participants, the overall results for sleep and work rhythms indicate a reasonable basis for adequate alertness to drive safely on a curvy road at night. Of course the most important function of the Driving Fitness Form was to assess individual fitness to drive on a given night.

As concerns the consumption of medications, drugs and alcohol, the results likewise indicate an adequate level of alertness for the sample as a whole. No participant took any prescription or non-prescription drug which comes with a warning not to operate a motor vehicle in the preceding 24 hours before the test drive. In the same 24 hours, $87 \%$ of the participants had consumed no alcohol at all, while $13 \%$ had consumed some alcohol. That alcohol consisted of a total of 10 glasses of wine and 15 glasses (shots) of liquor over all 126 drives for which forms were tallied. No single amount of alcohol was over 2 glasses or 2 shots for any given day. As concerns alcohol consumed in the last 12 hours, only one participant answered "yes" on one occasion. That occasion was about 8 hours prior to the drive and represented a relatively small quantity ( 2 shots of liquor). In this instance the participant felt alert enough to drive, and visual inspection and conversation confirmed the participant's fitness to drive. No participant was under the influence of any other substances that might impair her/his ability to safely operate a motor vehicle, and 98\% felt awake and alert enough to participate in the experiment "right now." On two occasions, a participant felt somewhat sleepy upon arrival to drive. In both of those cases, the participant was offered and drank coffee, after which she/he felt alert enough to participate. Visual inspection and conversation confirmed this conclusion.

The dedication and commitment of the participants was exceptional. For the 5 most critical days of the experiment, of the 80 scheduled drives, only one drive was missed and had to be rescheduled. The sample of research participants displayed considerable care to ensure their fitness to drive each night. Sleep and work rhythms were generally maintained in a manner compatible with the schedule of the experiment, and prescription medicine, alcohol and other substances were controlled in a responsible manner as well. The experiment was conducted without any incidents or crashes, and all participants expressed a willingness to assist in similar experiments in the future. 


\section{COMPARATIVE RESULTS}

The amount of sleep each participant had had before each drive was one of the most important factors in determining fitness to drive in the experiment. Figures 1 and 2 show the distribution of sleep durations across the entire experiment as separated by age (younger vs. older) and by sex (male vs. female), respectively. These data are presented only in the form of descriptive statistics concerning the particular sample of research participants in the present experiment. No relationships described are meant to characterize the population at large. The age comparison in Figure 1 reveals that the younger participants had a bimodal distribution with a smaller but still substantial peak at 3 hours, and a larger and maximum peak at 8 hours (mean $=6.63$ hours; std. dev. $=2.34$ hours). The older participants also had a bimodal distribution, but with a less substantial smaller peak at 2 hours, and a maximum peak at about 7 hours (mean $=6.65$ hours; std. dev. $=2.00$ hours).

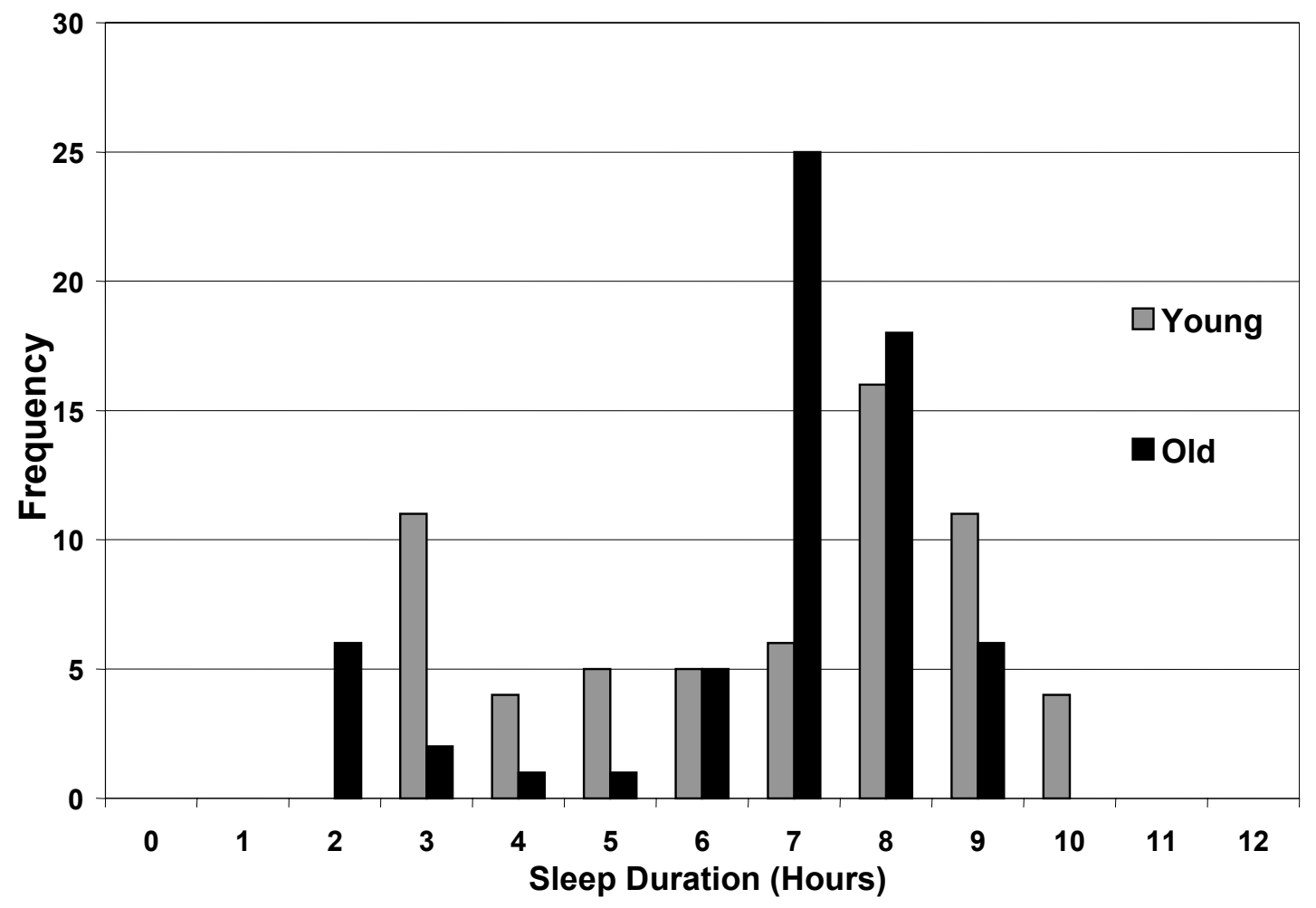

Figure 1. Distribution of sleep durations by age group

A somewhat different picture was obtained for sex. The sex comparison in Figure 2 reveals that the male participants had a bimodal distribution with a smaller peak at 3 hours and a larger maximum peak at 8 hours (mean $=6.40$ hours; std. dev. $=2.34$ hours). This pattern is similar to the younger age group in Figure 1. The female participants, however, had a skewed distribution toward the lower end, with a single major peak at 7 hours (mean $=6.95$ hours; std. dev. $=1.85$ hours). This pattern is somewhat different, and represents a skewed normal distribution of sleep durations. None of the differences between the pairs of mean sleep durations would be statistically significant, as is apparent by the relatively large standard deviations compared to the 
negligible mean differences. A more detailed examination of the individual data revealed that a few young males accounted for much of the bimodal shape and lower peaks in both figures.

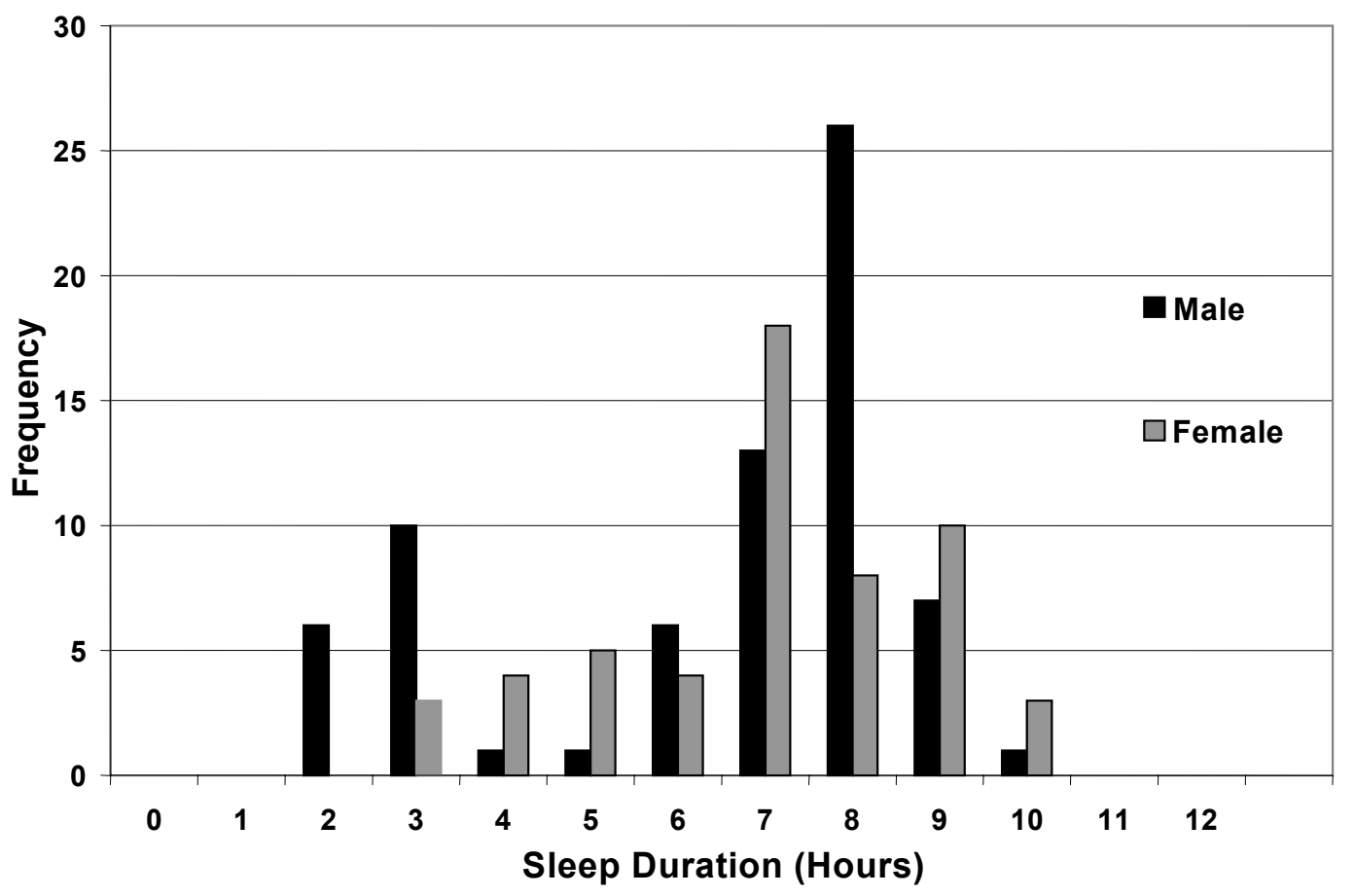

Figure 2. Distribution of sleep durations by sex

Thus, although the mean duration of sleep was the same between younger and older drivers, as well as between male and female drivers, the shapes of the distributions were somewhat different in the case of the female drivers. Due to the low number of participants in each category (only 8), no statistical tests were employed to determine the significance of any apparent differences in distribution shape for the particular sample. For the same reason of small sample size, no inferential statistics could be employed to characterize the population.

\section{CONCLUSIONS}

The objectives of this part of the research were met. An assessment instrument for fitness to drive was developed, this instrument was tested in a field experiment, data were collected on a sample of 16 research participants, and the sample of participants was characterized in terms of variables important to driving safety. The Driving Fitness Form met its primary objective, to ensure driving safety for participants and experimenters alike. All test drives were completed by all drivers on all nights without any incidents or crashes.

Minor improvements in the form were indicated for Question 7 concerning work patterns and for Question 16 concerning alcohol consumption. For Question 7, a "work/no work" discriminator question needs to be added up front to allow the retired people to drop through. For Question 16, further clarification between glasses and shots must be made, and a "none" response category needs to be added at the beginning of the quantity run. The next revision of the Driving Fitness Form will be directed at eliminating some of the confusion generated by these two questions. 
The overall successful implementation and testing of an assessment instrument for fitness to drive represents an important step in ensuring the safety of future FHWA field research experiments. Such precautions are especially important for nighttime field experiments, where sleep and work rhythms must be carefully balanced to ensure sufficient wakefulness and alertness to safely complete the required test drives.

\section{ACKNOWLEDGMENTS}

The authors wish to acknowledge the assistance of Jason Kennedy, Tiana Petit and Motoko Shimizu in reducing and analyzing the data for this paper. Jason Kennedy also recruited all 16 research participants in Pennsylvania. The authors also wish to acknowledge the vigilant service of David Harscheid, who was the Safety Observer for all 127 drives. Assisted by the Driving Fitness Form, Mr. Harscheid successfully ensured the safety of all who rode in the FHWA instrumented vehicle.

\section{REFERENCES}

Hartley, Laurence (Ed.) (1995). Fatigue and Driving: Driver Impairment, Driver Fatigue \& Driving Simulation. Bristol, PA, USA: Taylor \& Francis Inc.

Plain Language About Shiftwork. (1997). NIOSH (DHHS) Publication No. 97-145. 


\section{APPENDIX}

Participant/Staff Number

Date

Time AM PM

\section{Fatigue Assessment Form}

This form asks some basic questions about your state of fatigue and condition of alertness for participating in the experiment right now and for the next few hours.

1. What was the length of your last consolidated sleep period? ["av."=6.6] hours. ["sd"=2.2]

2. When did you go to bed? [NA ] AM PM

3. When did you wake up? [7:59] AM PM

4. What was the quality of your sleep? (Check one) Excellent [36\%] Good [62\%] Poor [2\%]

5. Is this your regular sleep pattern? Yes [37\%] No [63\%]

6. What were your work hours in the past 24 hours? Starting time [11:03] AM PM Ending

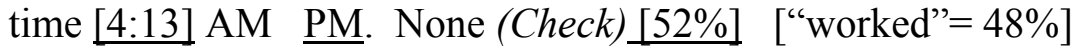

7. Are those your regular hours? Yes [34\%] No [29\%] NA [37\%]

8. Are you a shift worker? Yes [22\%] No [47\%] NA [31\%]

9. Have you crossed any time zones in the past 3 days? Yes [0\%] No [100\%]

10. In the past 24 hours have you taken any prescription or non-prescription drug which comes with a warning not to operate a motor vehicle? Yes [0\%] No $[100 \%]$

11. Give the approximate amount of alcohol you have consumed in the past 24 hours. ["some"= 13\%] ["none"=87\%]

Give the amount in terms of the standard glass size for that drink. Give the glasses of Beer [0], Wine [10], Fortified wine [0], Liquor [15], ["total glasses"]

12. Have you consumed any alcoholic beverage in the preceding 12 hours? Yes [1\%] No [99\%]

13. Are you under the influence of any other substances that might impair your ability to safely operate a motor vehicle? Yes [0\%] No [100\%]

14. Do you feel awake and alert enough to participate in this experiment right now? Yes [98\%] No [2\%] 